\title{
Evaluation of PPC / mBio Inc.'s Biochip Reader Integrated Circuit Reader System for Diagnosis of HIV / HCV Infection: Preliminary Method
}

\author{
Luciana Knop*1; Roberto Badaró1; Chris Myat ${ }^{2}$ \\ ${ }_{1}^{1}$ Health Institute of Technology - SENAI-CIMATEC; Salvador, BA, Brazil; \\ $2 \mathrm{~m} /$ Bio Diagnostics Inc. Precision Photonic Corporation, CO; USA
}

\begin{abstract}
The technological advances of recent decades in immunodiagnostic techniques have enabled the development of methods capable of detecting the antigen-antibody complex with high efficiency and reliability. However, the cost of such procedures has remained high; they are not easy to handle for an unskilled professional, nor provide immediate results for multiple samples. Precision Photonics Corporation (PPC), with $\mathrm{m} /$ Bio Inc. and in partnership with the University of San Diego (UCSD), USA, created an integrated circuit reader (Biochip Reader), which is low cost, fast and easy to handle, based on an optical systems and multiplex arrangements for the detection of biological multi-markers at the same time. This study aimed to evaluate the operation of the integrated circuit reader system (Biochip Reader), the protocols and the results of the multiplex tests for antibodies against HIV and HCV in partnership with the Federal University of Bahia (UFBA). We tested a total of 65 samples in the Biochip reader, and they showed $100 \%$ of sensitivity and specificity when compared to the results obtained by ELISA for HIV and HCV. Nevertheless, the protocols and the results obtained in Bahia presented slide instability, such as the formation of crystals and trehalose residues, unnecessary steps and manipulation of the slides. This fact led to changing the protocol and improving the prototype. However, more tests with new biomarkers are needed to validate the method.
\end{abstract}

Keywords: Biochip. Biosensor. HIV. HCV. Integrated circuit. Optical system. Fluorescence.

Infectious diseases have been one of the most significant public health problems in the world. Despite the diagnostic advances in medicine they still account for one in three deaths occurring worldwide [1]. In recent decades, technological progress has enabled the development of methods capable of detecting the antigen-antibody complex with high efficiency and reliability, through immunological techniques that reveal sensitivity to antigens (intradermal and percutaneous reactions), presence of antibodies or antigens in the body, (immunofluorescence, radioimmunoassay, enzymelinked immunosorbent assay, immunofluorimetry and chemiluminescence assays) [2]. Despite rapid tests, several immunodiagnostic tests still require specialized professionals to perform the

Received on 15 January 2019; revised 12 February 2019. Address for correspondence: Dr. Luciana Knop. Avenida Orlando Gomes, N. 1845, Piatã. Zip Code: 41650-010. Salvador, Bahia, Brazil. Phone: (+55 71) 3879-5501. E-mail: lbknop@hotmail.com.

J Bioeng. Biotech. Appl. Health 2019;2(1):3-14. C 2019 by SENAI CIMATEC. All rights reserved. procedures, an appropriate place for processing, specific materials and reagents and time for the determination of the results.

To create fast, cost-effective clinical diagnostic tests without the need for specialized professional, with little biological material, the Precision Photonics Corporation (PPC), in partnership with University of San Diego (UCSD), enhanced a biochip reader. This method was based in microarray-technology with an integrated circuit and photonic system for rapid and cost-effective diagnosis of numerous infectious diseases (PPC/ mBio Inc. - Biochip Reader). The Federal University of Bahia (UFBA), Brazil, joined this project to use this technique in the diagnosis of HIV, HCV, and related coinfections.

In this study, we present the results of Phase 2 of the project, with significant and promising results in the validation of biosensors for HIV and HCV. The Federal University of Bahia performed this Phase of the project in collaboration with Precision Photonics Corporation (PPC), mBio Diagnostic Inc., and the Division of Infectious Diseases of the University of San Diego (UCSD), California, 
USA, and the tests have been carried out in Brazil supported by Research Support Foundation of the State of Bahia (FAPESB).

\section{Development of Biochip Readers/Biosensors (Biochip Reader)}

\section{Naval Research Laboratory (NRL)}

The Multi-Analyte Array Biosensor (MAAB) was developed by the Naval Research Laboratory (NRL) of Washington (DC), USA, to detect and identify multiple analytes simultaneously [3]. The Biochip Reader is an electronic instrument developed for the detection of nanograms of toxins, allergens, proteins, bacteria, parasites or antibodies and antigens, using a minimal amount of biological material, without the need for specific processes $[4,5]$.

The NRL biosensor was designed using the microarray system and to be used with a laptop for fluid control, data analysis and storage [6]. Due to their selectivity and sensitivity, immunoassays were the first choice for the development of rapid identification methods using the fluorescencebased RL array biosensor [3].

\section{Precision Photonics Corporation}

Precision Photonics Corporation (PPC), USA, proposed a research and development program aimed at low-cost technology for the diagnosis of HIV and related coinfections. Due to the global nature of the disease and diagnostic products on the market, it was necessary to qualify the development tool outside the United States to assess its performance in a population with different conditions. Therefore, PPC and the corporate division of mBio Diagnostic adapted the biosensor of NRL arrangements and successfully developed prototypes of a low-cost diagnostic tool for infectious diseases. A biochip (consumer disposable) - containing the analytic for recognition; the transducer (non-disposable); and the reader (non-disposable) are the basement of this equipment (Figure 1).
Figure 1. Biochip Reader Model.

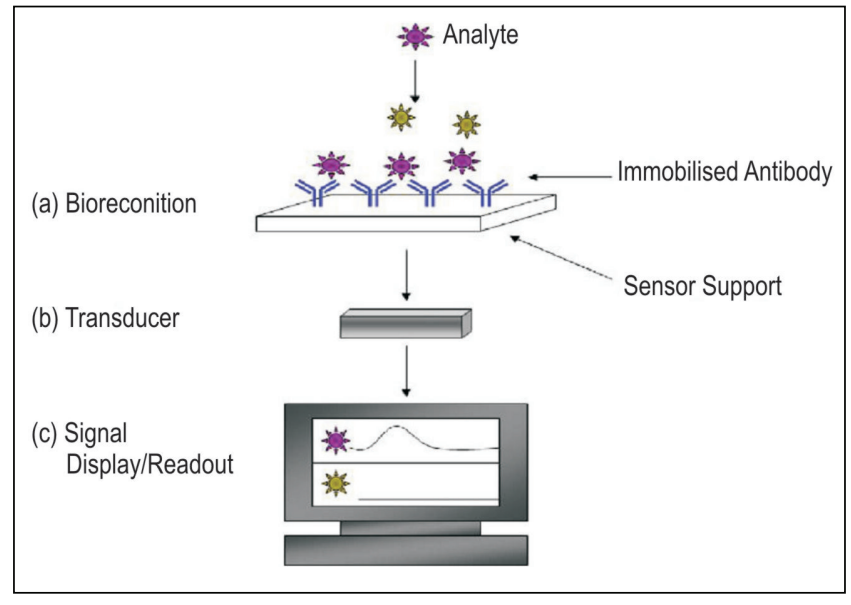

\section{Development of the Biosensor by PPC}

The biochip's PPC reader is a fluorescencebased laser optical detection system and uses a laser diode, and a CMOS or CCD toner comprised the source and the detector. The equipment consists of a laptop, a multi-pathogens biosensor (reader) to detect analytes at one time, a separate interface to avoid contamination (Figure 2a - details in Figure 3 ) and the 'slides' in the form of microarray strips, previously sensitized (Figure 2b). It is simple, portable, easy-to-operate equipment that requires minimal sample preparation and low-power handling, and it is appropriate for use in rural areas and districts with poor socioeconomic conditions.

\section{Methods of Biochips Units (Diagnostic Cards)}

The biochip (diagnostic card) is a miniaturized plastic strip, arranged in microarrays, with the presence of negative and positive controls and sensitized with the antigens of HIV and HCV. The preparation of these strips specifically for these target pathogens occurred in Phase 1 in the United States.

\section{Preparation of Biochips to Phase II}

The samples were placed in closed containers and pumped directly into the chips at Precision Photonics Corporation (PPC), USA. Thus, the chips (slide/trips) (Figure 2b) presented specific areas containing concentrations of antigens by enzymelinked immunoassays, such as the indirect ELISA 
Figure 2. (a) Biochip Reader; (b) slides (trips) in micro-arrays (biochip) previously previamente sensitized.

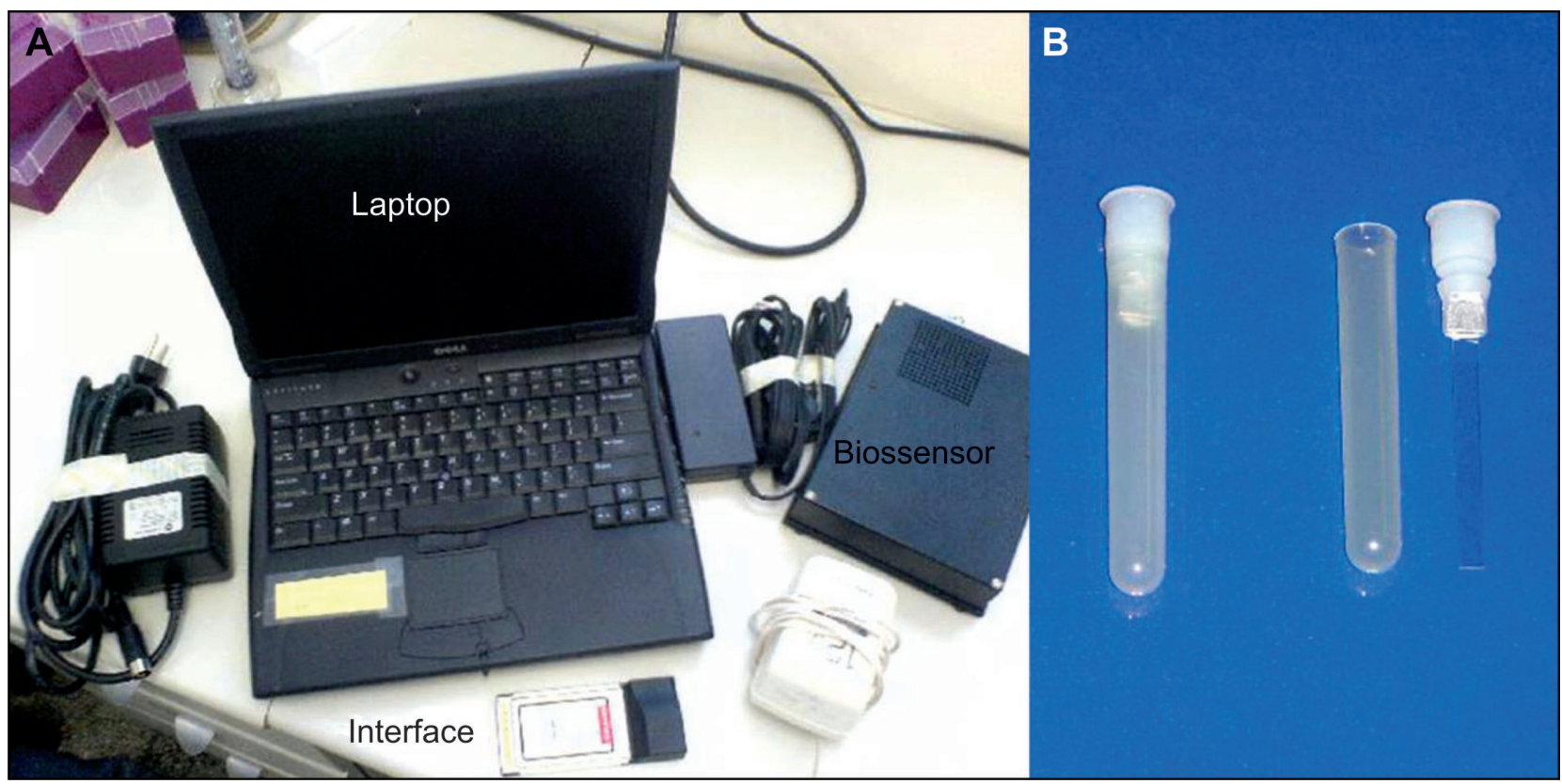

Figure 3. Biosensor detail.

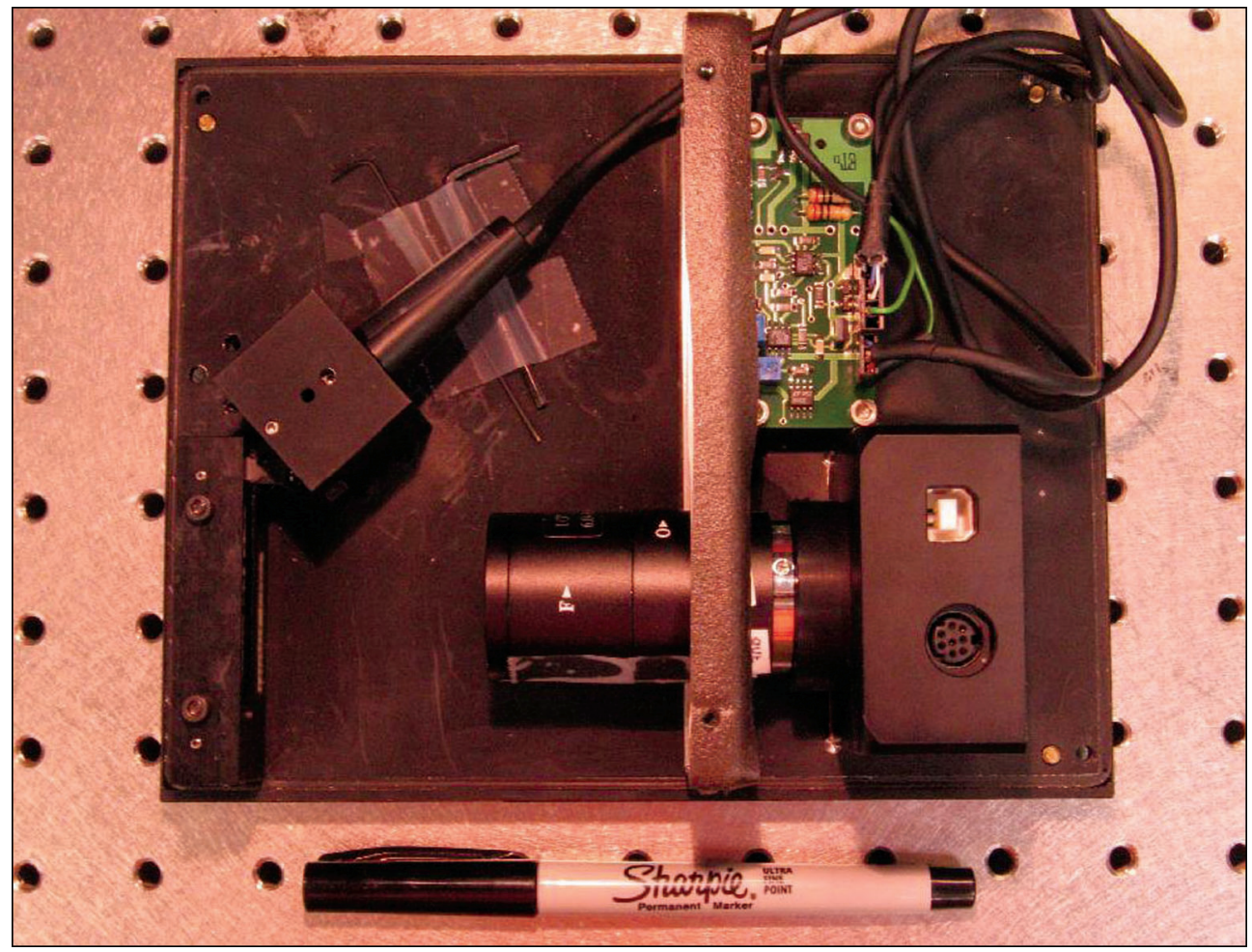


technique. Subsequently, each pathogen was identified electronically and placed within a particular location where the pumping of the sample was directed (Biodot-Arrayer). In this stage of construction, doseresponse curves were prepared for each pathogen to compare the results obtained in Phase I.

\section{The Study}

The Laboratory of Tropical Diseases of the University Complex Prof. Edgard Santos (HUPES) at the Federal University of Bahia (UFBA) conducted this study. It consisted of the evaluation and application of the material sent by PPC / mBio, which include: strips/slides already previously sensitized and blocked in the USA (Biochip Reader with BCRanalysis software and runcamera.exe installed on a laptop), protocols 1 and 2; using the samples (sera/plasma) collected and data provided from HUPES patients as well as materials and reagents.

We selected 65 sera/plasma from patients positive and negative for HIV, HCV, and HTLV and related coinfection. We chose randomly 52 old sera from the Tropical Diseases Laboratory, following protocol 1 (Figure 4); and 13 (7 sera and 6 plasma) during protocol 2 (Figure 5).

This project was approved by the National Committee for Research Ethics (CONEP) of UFBA on June 20, 2006, under number 60/2006 and is still in force. The classification of this article, according to the Classification System for Journal Articles of the Journal of Economic Literature (JEL), is I1.

\section{Protocol and Procedures Tests}

PPC/mBio (Figures 4 and 5) sent two protocols for the tests carried out in Bahia. Protocol 1 (Figure 4) showed antigens for the HCV core and HIV gp41 protein viral envelope. Protocol 2 (Figure 5), in addition to the antigens referred to in the previous protocol, the antigens of the HIV gp120 proteins, HCVNS3, and the reduction of a spots grid for both positive and negative control were inserted, and the protocol included a large number of spots for human IgG control. Besides, spots containing HIV gp41 antigen were enlarged.
Regarding the reagents and procedures adopted, the two protocols were identical.

The protocols provided the procedures: (1) strip washing and new blocking; (2) incubation with the human serum samples; (3) incubation with labeled antibody; (4) rinsing, drying and image detection on the reader (Biochip Reader).

We obtained human serum samples with HIV / HCV (+/-), (-/+) and (-/-) from the serum bank of the Tropical Medicine Laboratory at the Federal University of Bahia. The blocking buffer used was commercial Pierce buffer, mixed in the ratio of $1: 1$ with the Tris-EDTA buffer. Detection of the labeled antibody used was anti-human IgG Alexa 647 (Invitrogen / Molecular Probes).

Data Analysis and Determination of Exposure Time

BCRanalysis performed the Data analysis by the software of the Biochip Reader, which analyzed the image-points with specific programming. This programme probes the intensities of the spots for HIV and $\mathrm{HCV}$ were relative to the positive and negative controls in the same slide. PPC/mBio defined the cut-off in the procedures forwarded to designate the positive or negative result, using the pre-programming of $<255 \mathrm{~ms}$, without the use of background subtraction and images with 1280x1024 pixels.

\section{$\underline{\text { HIV/HCV Sensitized Slides }}$}

Patients' sera were identified according to positivity or negativity alone or with coinfection, as described in Table 1.

Table 1. Tested samples of HIV, HCV, HTLV negative and positive patients and coinfection related.

\begin{tabular}{lccccc}
\hline Slides & \multicolumn{5}{c}{ Patients (N=65) } \\
\cline { 2 - 6 } /Infection & HIV+ & HIV- & HCV+ & HCV- & Total \\
\hline HIV* & 25 & 0 & 0 & 25 & 25 \\
HCV* $^{*}$ & 0 & 13 & 13 & 0 & 13 \\
HTLV* & 0 & 6 & 0 & 6 & 6 \\
HIV+HCV & 9 & 0 & 9 & 0 & 9 \\
HIV+HTLV & 6 & 0 & 0 & 6 & 6 \\
HCV+HTLV & 0 & 6 & 6 & 0 & 6 \\
Subtotal & $40 * *$ & $25 * *$ & $25 * *$ & $37 * *$ & $65 * * *$ \\
* Monoinfected patients. ** Total of patients with or without \\
infection. *** Overall of patients.
\end{tabular}


Figure 4. PPC/mBio Protocol 1. Disposition of HIV gp41 protein viral envelope, HCV core antigens, and positive and negative controls on the slides.

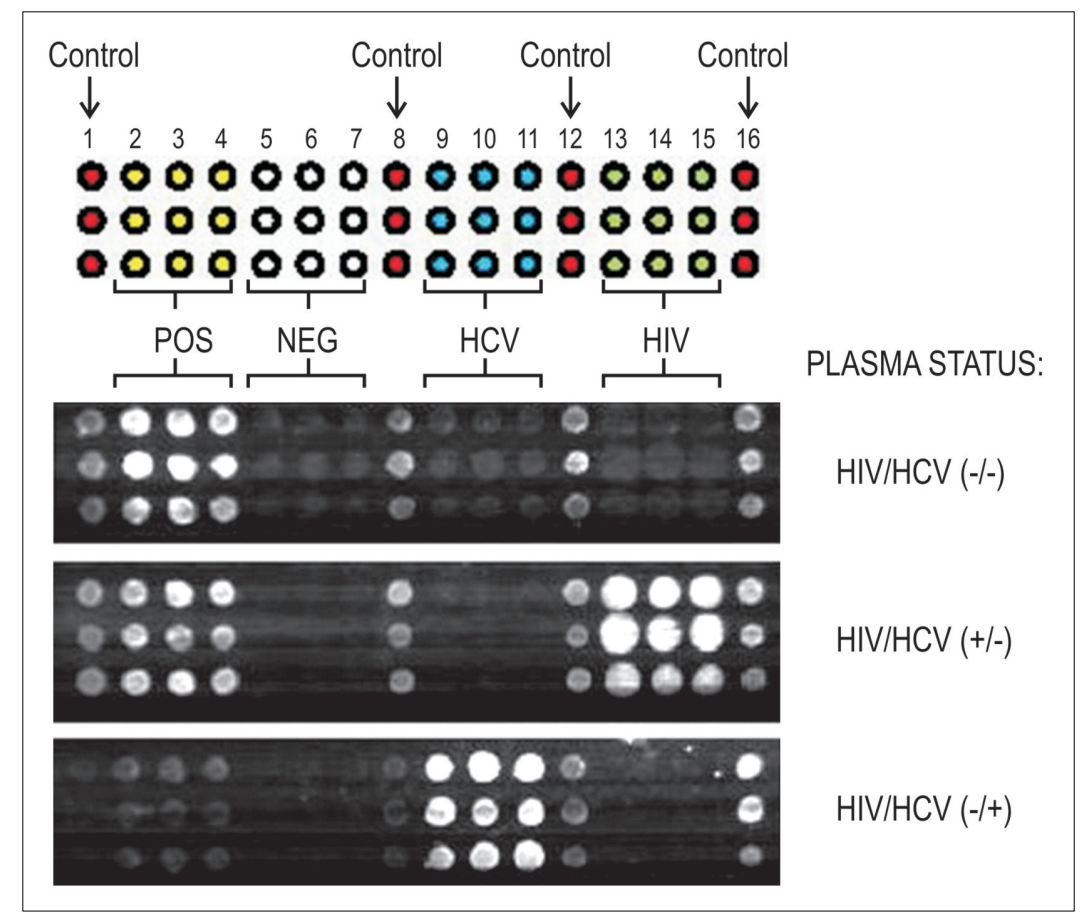

Figure 5. PPC/mBio Protocol 2. Disposition of gp41 and gp120 HIV antigens, core and Nns3HCV antigens, and positive and negative controls on the slides.

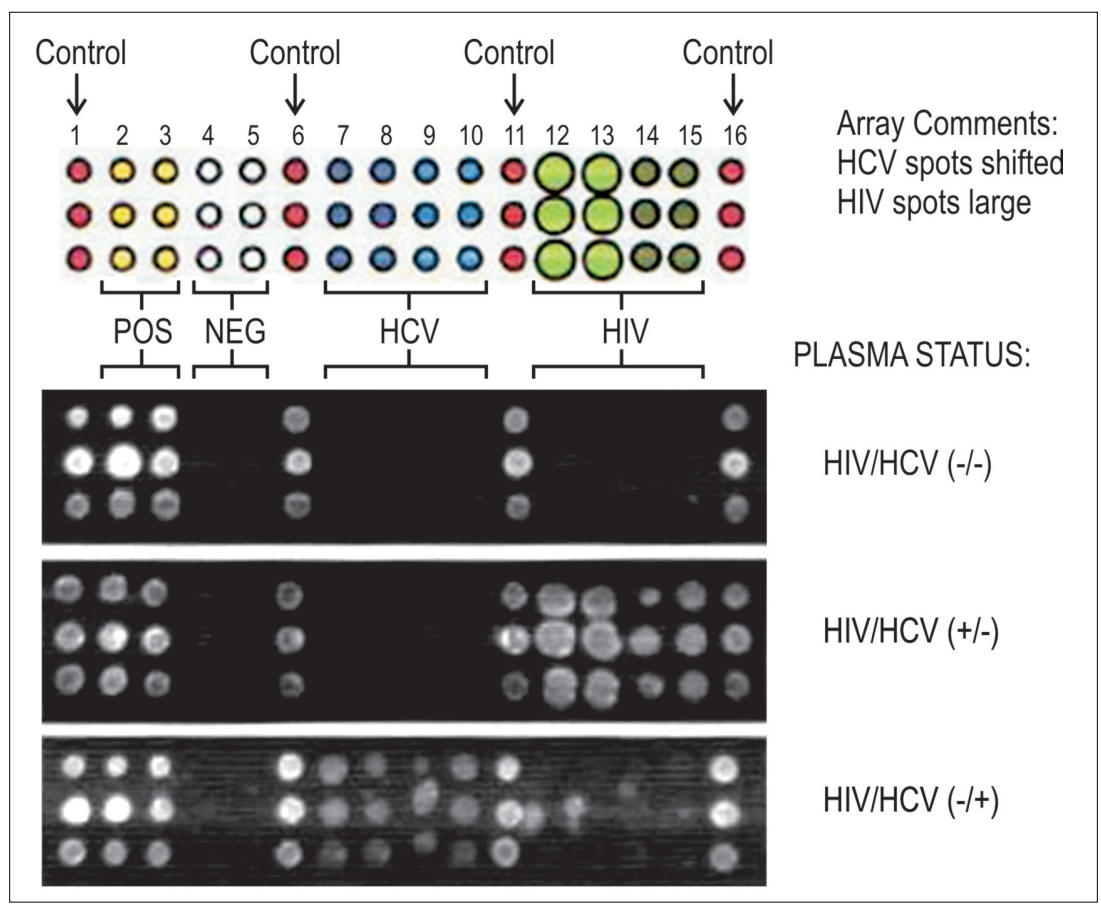


Figure 6. HIV/HTLV positive patient. Slide shows HIV positive patient.

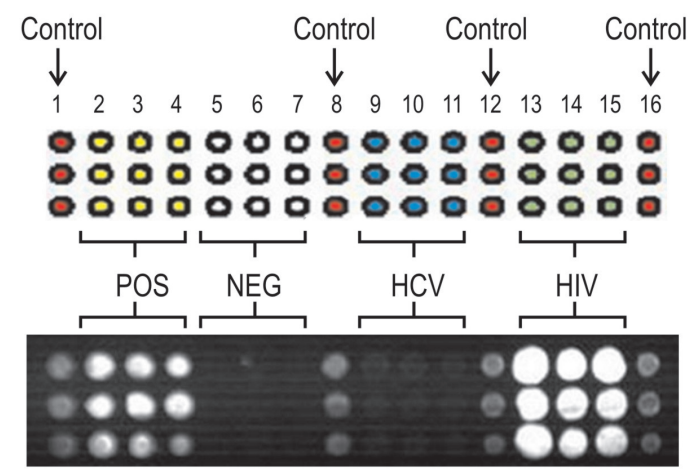

Figure 7. HIV/HCV/HTLV negative patient. Slide shows HIV/HCV negative patient.

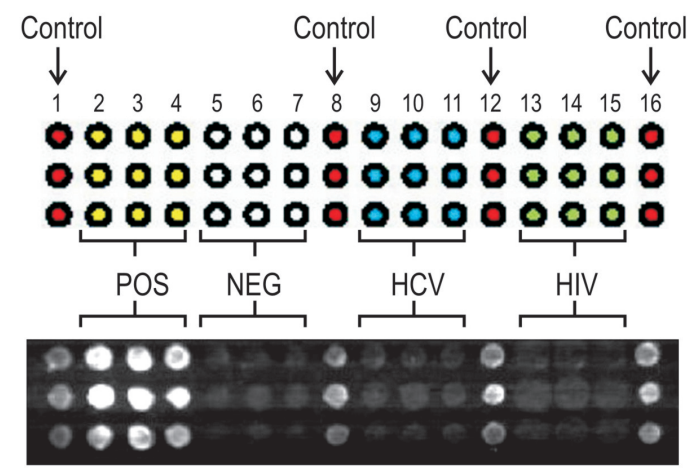

Figure 8. HIV/HCV positive and HTLV negative patient. Slide shows HIV/HCV positive patient.
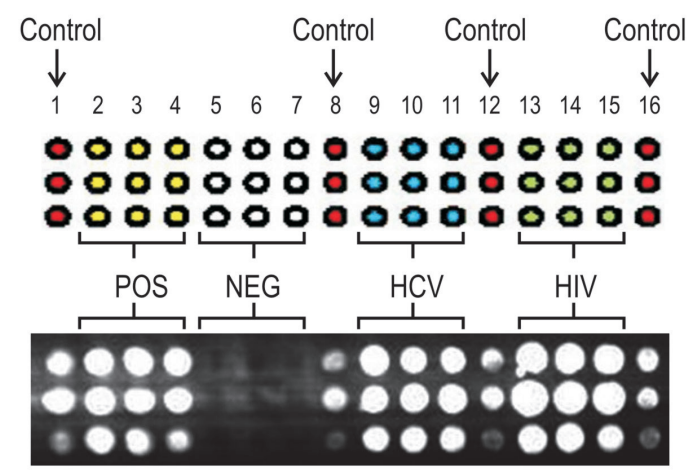

Figure 9. HIV/HCV/HTLV positive patient. Slide show HIV/HCV positive patient.

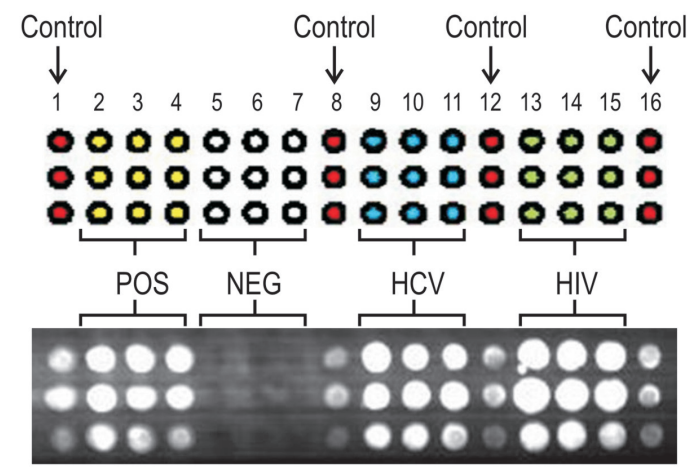


Figure 10. HCV/HTLV positive patient. Slide shows HIV negative and HCV positive patient.

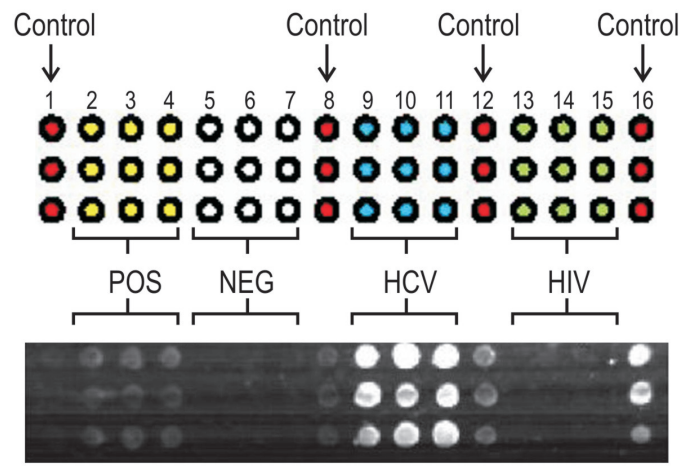

The results obtained showed sensitivity and specificity of $100 \%$ when correlated with previously known results from the serum file of HUPES, which used the ELISA method. In all HTLV positive patients tested, there was no cross-reaction or interference noise in areas sensitized with HIV and HCV specific antigens and controls (Figure 6). The slides 1 and 5 were not blocked during protocol as determined by the procedure; however, this did not change the final results. In the remaining 47 samples, protocol 1 followed regularly, and the result was consistent with the results previously known to the patients (Figures 7 to 10).
Evaluation of Protocols

$\mathrm{PPC} / \mathrm{mBio}$ submitted 38 slides for testing following the protocol 2 . Table 2 summarizes the events with this material.

Thus, of the 38 slides sent, only 13 slides were tested according to the second protocol, which was also compatible with ELISA results, performed shortly after collection from the patients. The lack of blockade in 5 slides during the application of protocol 2 also did not affect the final results (Figures 11a, 11b, 11c - the same patient - and 12a, $12 \mathrm{~b}$ - other patients). Figures 13 to 16 present the results of 13 slides tested according to protocol 2.

Table 2. Occurrences with slides sent by PPC / mBio (protocol 2).

\begin{tabular}{lc}
\hline Events & Slides (N) \\
\hline Lack of slide attachment in Biochip Reader camera & 10 \\
Contamination by fungi & 8 \\
No operation of the device at the time of reading (no signal, background) & 7 \\
\hline Total & $\mathbf{2 5}$
\end{tabular}

Figure 11a. Slide shows HIV positive patient. Lack of blockade the slide during procedure. Signal to gp120 and weak positive controls.

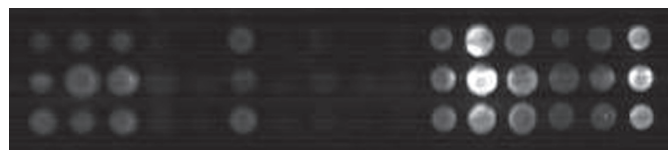


Figure 11b. Slide shows HIV positive patient. Correct use of the protocol (blockade). Signal to gp120 and weak positive controls.

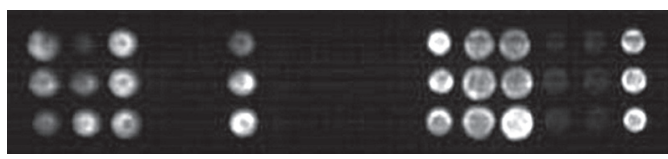

Figure 11c. Slide shows HIV positive patient. Correct use of the protocol (blockade). Normal signal to gp 120.

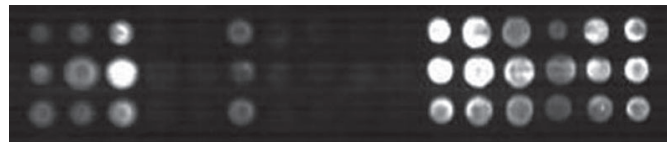

Figure 12a. Slide shows HIV positive patient. Lack of blockade the slide during procedure. Weak signal to gp120.

\begin{tabular}{|c|c|c|}
\hline $\begin{array}{l}0.0 \\
0 \% \\
0\end{array}$ & $\begin{array}{l}0 \\
0\end{array}$ & $\begin{array}{l}0 \% \\
00 \\
\circ 0\end{array}$ \\
\hline
\end{tabular}

Figure 12b. Slide shows HIV positive patient. Correct use of the protocol (blockade). Normal signal to gp120.

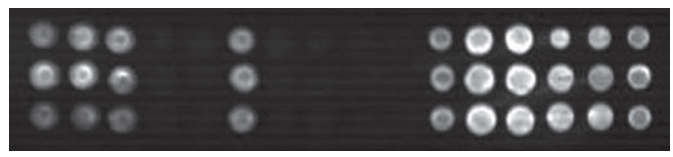

$\underline{\text { Statistical Analysis }}$

Data analysis was performed by BCRanalysis, the software of the Biochip Reader, which analyzed the image points with pre-specific programming by $\mathrm{PPC} / \mathrm{mBio}$, and SPSS Software to calculate median, mean and mode.

\section{Time of Exposure (protocol 2)}

Tests with the exposure time of $50 \mathrm{~ms}$ obtained the best results (Table 3), this being the time of exposure most indicated during this experiment.
Table 3. Time of exposure of slides - mean and mode.

\begin{tabular}{lcc}
\multicolumn{2}{l}{ Time of exposure $(\mathbf{m s}) *$} \\
\hline $\mathrm{N}$ & Valid & 13 \\
& Not valid & 0 \\
Mean & & 57.92 \\
Mode & & 50 \\
\hline \multicolumn{2}{l}{ * } & SPSS software.
\end{tabular}


Figure 13. HIV positive patient. Weak signal to gp120.

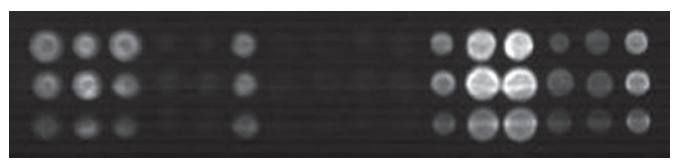

Figure 14. HIV positive patient (coinfected with cytomegalovirus). Weak signal to gp120.

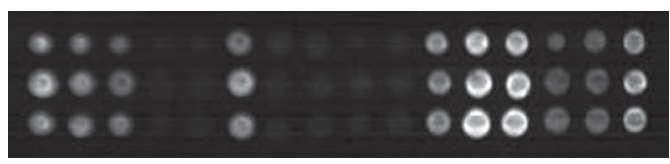

Figure 15. HIV positive patient with disseminated tuberculosis. Backgroung to negative controls and $\mathrm{HCV}$.

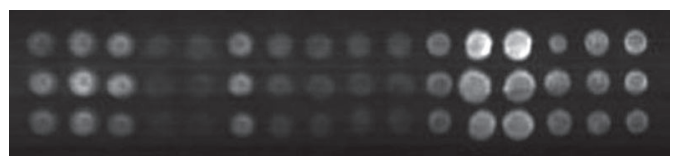

Figure 16. HIV/HCV/HTLV positive patient. Weah signal to gp120 and background to HCV antigens.

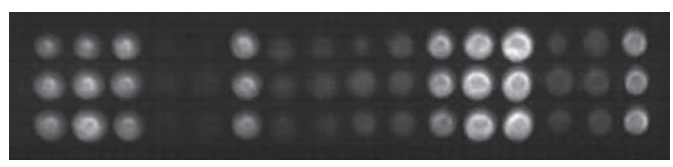

The advances in miniaturization allowed the development of portable systems, electronic and digital components. This development enabled the discovery of the microarray system with the power of simultaneous analysis of thousands of molecules at the same time $[3,8]$, and the improvement of the biosensor. In this study, we describe a Biochip Reader platform for diagnosis that provides a single-sample marker analysis at the same time. The method consists of using a disposable cutting slide integrated with a fluorescence reader based on planar and multimode wave conductors capable of analyzing different tests for detection of specific antibodies (HIV/HCV) at the same time. The sensitivity and specificity were of $100 \%$, without cross-reaction with other known pathogens (HTLV and cytomegalovirus). The tests revealed the ability of the system to maintain the antigen/antibody reaction specific to the researched condition. We also analyzed all the technical steps and procedures, manuals and protocols sent by $\mathrm{PPC} / \mathrm{mBio}$ to obtain the results. 
The results showed the excellent reliability of the system. However, the poor quality of the slide manufactured in the USA may have interfered and affected the experiment carried out in Bahia (UFBA) due to the presence of weak signals, backgrounds in slides or no sharp image in the signal emission, which possibly did not allow good antigen-antibody binding and the action of the fluorophore.

This study emphasized the importance of the quality of the material to be chosen as a substrate for the bioreactor, such as the polishing of the slides [15-16]. Battison (2001) [17] and Vo-Dihn $(1998 ; 2000)$ [18,19] described that substrate polishing is fundamental for good wave conduction and signal emission. As a result, in our study, the poor quality of the slide due to poor polishing of the glass might have impaired and prevented the conduction of the evanescent wave adequately, which was decisive for the presentation of the weak controls, the presence of background and low signal of the antigens for HCV during the use of protocol 2. So, the polishing problems may be related to the imprinting (PPC), causing "dragging" of the molecules during the washing process and subsequently protocol procedures [20].

Also, Monk (2004) [20] and Grace (2011) [16] affirm that for optical systems that use planar wave conductors, they must have their substrate properly polished otherwise they will be unable to conduct the wave and create random or inappropriate signals. According to Feldstein (1999) [21], a fluorescence biosensor, based on planar wave conductors, will work correctly, if the surface of the biochip base (glass, silica or other suitable material) is appropriately polished. This condition may be one of the factors that left all the weak positive controls in the first position, according to protocol 2.

Another problem was the fungus contamination that could occur due to the quality of the slides, transport, and excessive humidity present in our environment or inadequate storage. However, according to Vo-Dihn (2000) [22] and Rowe (2011) [23] biosensors should not have many specifications regarding storage and handling, since the intention is to create a portable and easy handle system.
The slides showed crystal formation and an excess of trehalose, which may be caused by the high temperatures in Brazil [24], which brings the importance of choosing the substrate for the printing of biochips, polishing and pre-blocking with appropriate washes so as not to permit the residue of sugars, which could interfere in the results (backgrounds, inability to signal and read) [7,25].

Notwithstanding, the extremely positive point is that, despite the occurrences in the signals, there was a sensitivity of $100 \%$ in the samples analyzed. Besides, the sera used during the procedures of protocol 1 were old, and this does not seem to change the results. Plasma and serum were also used during the process with protocol 2, and there was no evidence of any change in the results obtained compared to the results using the ELISA method performed concomitantly during this experiment.

The use of two protocols for the tests performed in Bahia occurred due to the emission of more stronger signals in the HIV go 41 region, presenting a "ballooning" of the area for HIV gp41 during the experiment with protocol 1 (Figures 4, 9 and 10). This phenomenon raises doubts as to the right binding in the biomolecules adhered to the spots, because of the overflow which occurred and the bond could occur outside the space previously printed, emitting stronger signals ( $>255 \mathrm{~ms})$, impairing the reading of the slides [26]. This event occurred due to the size of the protein, but it was adjusted in the second protocol to remain no doubt about the results.

The pre-blocking and printing on biomolecule integrated circuits are punctual phases for biochip formation according to Lochhead (2008) [26], Grace (2011) [16] and Macbeath (2000) [27]. Our results showed an excellent pre-blocking performed by $\mathrm{PPC} / \mathrm{mBio}$, since the changes made in the procedures, without the buffering of the slides, did not cause differences in the final result.

The time of exposure is one of the determining factors for the knowledge of sensitivity and specificity in multi-strand-based optical biosensor tests since the amount of $\mathrm{ms}$ is fundamental to the reliability of the experiments [26]. Sometimes it 
is difficult in normalizing the signal of one strand with the sign of another strand in the biosensors due to the differences between optical fibers. This issue is related to the variation of the exposure time to which each fiber is submitted $[25,28,29]$. As each antibody has a specific intrinsic fluorescence, the fluorophores are inserted to stabilize the signal during the analysis $[16,30]$. However, if the exposure time is not "normalized," or preprogrammed on the optical substrate, there will be no stability about exposure time, and this could lead to significant variations in the readings for the same bioreactor [11,12,28,29,31].

So, the time of exposure time was also a critical factor observed in the experiment, since the tests performed with protocol 2 were well below the 255 ms stipulated as a cut-off for reading. During the tests with protocol 2, the best exposure time was $50 \mathrm{~ms}$, without the use of background subtraction and images with $1280 \times 1024$ pixels.

The results obtained for the exposure time also could be related to the limitation of mass transfer instead of a fluorescence transduction signal. The mass transfer has long been recognized as a limitation in this type of microarray system [30,31], which may have led to signal weakening, poor readability, or low exposure time for microarrays in the performance of protocol 2.

Furthermore, data analysis showed that each antigen would require tests at different concentrations in the same blocker and patient serum samples and their dilutions to precisely determine which antigen, blocker, and dilution concentrations of serum samples the experiment should have. It will allow better sensitivity and specificity detection in this multi-analyte format.

Phase II of the study showed good sensitivity and specificity of the system despite the problems presented. More experiments and further improvements are necessary for multi-pathogen detection, which is fast, cost-effective and does not require professional ability to handle the system.

The experiments performed in Brazil showed satisfactory results regarding the performance of the integrated circuit reader system (Biochip Reader) (the PPC/mBio Inc.) in the diagnosis for antibodies against $\mathrm{HIV}$ and $\mathrm{HCV}$, with a sensitivity and specificity of $100 \%$. Nevertheless, adjustments are still necessary to make PPC/mBio's Biochip Reader an efficient, low-cost, portable and easy-to-handle tool. These improvements include larger sampling volume, technical development (slides/trips), optimization of protocols, and standardization of exposure time without large intervals.

\section{Future Perspectives}

The University of San Diego and PPC m/Bio updated the model as a result of the tests carried out in Brazil. This new prototype has more resistant tools; technical improvement of the biosensor, bioreactors, and biochip; and innovative design of slides; better polishing of antigen-specific fixation areas; new lens models and optical filters; fluid channel system more suitable for mass transfer; panel expansion of controls; and a new protocol. Also, the software was adequate and easy to use and handle, with a higher degree of automation and less possibility of manipulation by the user (Figure 17).

PPC and $\mathrm{m} /$ Bio sent the new prototype to Brazil (Figure 18) with the original slides, protocols and faster procedures for validation of tests for HIV, HCV, HBV, T. pallidum and T. gondii. The activities are in progress, and promising results would be obtained for the final stage of the validation of the tests.

Figura 17. A. Model of the wave driver and the new slide. B. Figure of the new Biochip Reader.

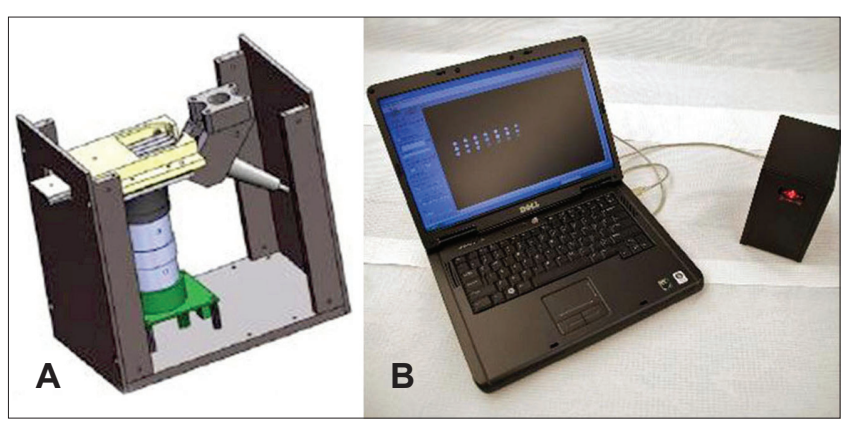


Figure 18. Protocol for using the new slide.

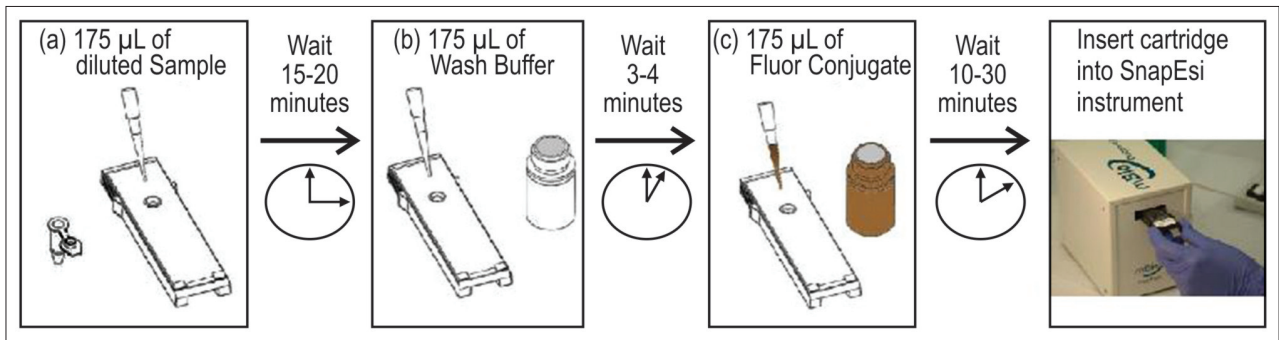

\section{References}

1. WHO. Infectious diseases in all world. Bulletin of the World Health Organization, 2016; v 9, n. 8:545-620.

2. Vaz, A., Takei, K., Bueno, E.C. Imunoensaios: Fundamentos e Aplicações. Rio de Janeiro: Guanabara Koogan, Capítulo 2, 2007.

3. Taitt, C.R. et al. A portable array biosensor for detecting multiple analytes in complex samples. Microb Ecol, 2004;2:175-185.

4. Bakaltcheva, I.B., Shriver-Lake, L.C.; Ligler, F.S. A fiber optic biosensor for multianalyte detection: importance of preventing fluorophore aggregation. Sensors and Actuators B-Chemical, 1998;1-3:46-51.

5. Bakaltcheva, I.B., Shriver-Lake, L.C.; Ligler, F.S. Multianalyte explosive detection using a fiber optic biosensor. Analytica Chimica Acta, 1999;1-2:13-20.

6. Ligler, F.S. et al. The array biosensor: portable, automated systems. Anal Sci, 2007;1:5-10.

7. Albert, K.J. et al. Cross-reactive chemical sensor arrays. Chem Rev, 2010;7:.2595-2626.

8. Narang, U. et al. Multianalyte detection using a capillarybased flow immunosensor. Analytical Biochemistry, 1998;1:13-19.

9. Sapsford, K.E. et al. Kinetics of antigen binding to arrays of antibodies in different sized spots. Analytical Chemistry, 2001;73:5518-5524.

10. Sapsford, K.E. et al. Array biosensor for homeland defense. Abstracts of Papers of the American Chemical Society, 2003;U107.

11. Ligler, F.S. et al. Integrating waveguide biosensor. Analytical Chemistry, 2002;3(713-719).

12. Ligler, F.S. et al. Array biosensor for detection of toxins. Anal Bioanal Chem, 2003;(3): 469-477.

13. Rippel, R.A., Seifalian, A.M. Gold revolution - gold nanoparticles for modern medicine and surgery. J Nanosci Nanotechnol, 2011;May;11(5):3740-8.

14. Pantic, I. Nanoparticles and modulation of immune responses. Sci Prog., 2011;94(Pt 1):97-107.

15. Cha,T.G. et al. Optical nanosensor architecture for cellsignaling molecules using DNA aptamer-coated carbon nanotubes. ACS Nano, 2011;May 24;5(5):4236-44.

16. Grace, K.; Swanson, B. Optical Biosensor Reagentless (ROB). Los Alamos National Laboratory, Los Alamos Biosensor Team, 2011.
17. Battison, F.M. et al. A chemical sensor based on a microfabricated cantilever array with simultaneous resonance-frequency and bending readout. Sens Actuators 2001;77:122131.

18. Vo-Dinh, T. et al. Optical nanosensors for detecting proteins and biomarkers in individual living cells. Clin Diagn Lab Immunol,1998 September;5(5):609-612.

19. Vo-Dinh, T. et al. DNA biochip using a phototransistor integrated circuit.Anal Chem 2000Jan 15;71(2):358-63.

20. Monk, D.J., Walt, D.R. Optical fiber-based biosensors. Anal Bioanal Chem 2004;379:931-945.

21. Feldstein, M.J. et al. Array biosensor: optical and fluidics systems. Journal of Biomedical Microdevices, 1999;1:139-153.

22. Vo-Dinh, T. et al. Biosensors and biochips: advances in biological and medical diagnostics. J Anal Chem 2001;366:540-551.

23. Rowe, C.A. et al. An array immunosensor for simultaneous detection of clinical analytes. Anal Chem, 2011;2:433-439.

24. Ziegler, C. Cantilever-based biosensors. Anal Bioanal Chem 2004;379:946-959.

25. Herron, J.N. et al. Fluorescence Sensors and Biosensors. Taylor \& Francis CRC Press, Boca Raton, FL, 2005:283332.

26. Lochhead, M.J., Delaney, M., Radka, S. et al. Low Cost, Multiplexed Biosensor Technology for Simultaneous Analysis of HIV and Common Co-Infections. AACC Annual Meeting, Vol. Washington, D.C., 2008.

27. Macbeath, G., Schreiber, S.L. Printing proteins as microarrays for high-throughput function determination. Science, 2000;5485:1760-1763.

28. Herron, J.N. et al. Waveguide immunosensor with coating chemistry providing enhanced sensitivity. US Patent, 1996;5(512): 492.

29. Herron, J.N. et al. Planar Waveguide Biosensors for Point-of-Care Clinical and Molecular Diagnostics. In: Thompson RB, ed. Fluorescence Sensors and Biosensors, Vol. Boca Raton, FL: CRC Press Taylor \& Francis Group, 2006.

30. Lee, Y.E., Smith, R., Kopelman, R. Nanoparticle PEBBLE sensors in live cells and in vivo. Annu Rev Anal Chem (Palo Alto Calif), 2009;2:57-76.

31. Kenneth, E. et al. Biomedical Nanostructures JohnWiley\&Sons, Inc. 2010; Chapter 17. 